\title{
Qualidade de vida e síndrome metabólica em mulheres brasilei- ras: análise da correlação com a aptidão aeróbia e a força mus- cular
}

\author{
Quality of life and metabolic syndrome in Brazilian women: analysis of \\ the correlation with aerobic fitness and muscle strength
}

Tatiane Gomes Teixeira1*, Ramires Alsamir Tibana ${ }^{1}$, Dahan da Cunha Nascimento ${ }^{1}$, Renato André Sousa da Silva ${ }^{1}$, Jeeser Alves de Almeida ${ }^{1}$, Sandor Balsamo ${ }^{2}$, Fabricio Azevedo Voltarelli ${ }^{3}$, Jonato Prestes ${ }^{1}$

ARTIGO ORIGINAL | ORIGINAL ARTICLE

RESUMO

A síndrome metabólica (SM) é definida pela presença de, ao menos três, dentre cinco fatores de risco cardiovascular: hiperglicemia, resistência à insulina, obesidade, dislipidemia e pressão arterial elevada. Embora portadores de SM apresentem baixa aptidão física e pior qualidade de vida (QV), quando comparado aos indivíduos sem SM, ainda não foram investigadas as correlações entre a aptidão física e a QV em mulheres com SM. O objetivo do presente estudo foi comparar a QV, força muscular e aptidão aeróbia, bem como analisar a correlação entre a QV e a aptidão física de mulheres com e sem SM. Participaram do estudo 49 mulheres, 21 com SM $(33.5 \pm 9.1$ anos $)$ e 28 sem SM (32.3 \pm 7.9 anos). Para avaliação da QV, aptidão aeróbia e força muscular foram utilizados, respectivamente, o questionário Short Form Health Survey 36 (SF-36), o teste de caminhada de 6 minutos (6-C) e o teste de preensão manual. As mulheres com SM apresentaram pior QV, percorreram menor distância no 6-C e obtiveram menor força muscular relativa. Foi observada correlação positiva entre a aptidão aeróbia e quatro domínios da QV: capacidade funcional, limitação por aspectos físicos, dor e aspectos sociais da funcionalidade. Mulheres com SM apresentam menor de força muscular e aptidão aeróbia, bem como piores escores de QV no estado de saúde geral, capacidade funcional, dor no corpo, limitação por aspectos emocionais, aspectos sociais da funcionalidade e limitação por aspectos físicos quando comparado com mulheres sem SM.

Palavras-chaves: Síndrome Metabólica, Aptidão Física, Qualidade de Vida, Doenças Cardiovasculares

ABSTRACT
The metabolic syndrome (MetS) is defined as the presence of at least three out of five cardiovascular risk factors: hiperglicemia, insulin resistance, abdominal obesity, dyslipidemia and elevated blood pressure. Subjects with MetS still present worse perception of quality of life (QL) and low physical fitness as compared with subjects without MetS. However, correlations between physical fitness and QL have not been investigated in subjects with MetS. The aim of the present study was to compare the QL, muscle strength and aerobic fitness, as well as to analyse the correlatation between the QL and physical fitness in women with and without MetS. Forty-nine women, 21 with MetS (33.5 \pm 9.1 years) and 28 without MetS (32.3 \pm 7.9 years) were studied. Perception of QL, physical fitness and muscle strength were evaluated by the Short Form Health Survey 36 (SF-36), the distance covered in the 6 minute walk test (6MWT), and the handgrip test, respectively. Women with MetS presented worse perception of QL, covered a lower distance during the 6MWT and exhibited lower relative muscle strength. Additionally there was a positive correlation of aerobic fitness with four domains of QL: functional capacity, limitation by physical aspect, pain and social aspects. Women with MetS present lower muscle strength and aerobic fitness, as well as lower QL scores for the general health state, functional capacity, body pain, limitation by emotional aspects, social aspects of functionality and limitation by physical aspects as compared with women without MetS.

Keywords: Metabolic Syndrome, Physical Fitness, Quality of Life, Cardiovascular Diseases

Artigo recebido a 24.02.2014; Aceite a 09.07.2014

${ }^{1}$ Faculdade de Educação Física da Universidade Católica de Brasília, Brasília, Brasil

${ }^{2}$ Centro Universitário Euro Americano (UNIEURO), Departamento de Educação Física, Brasília, Brasil

${ }^{3}$ Universidade Federal de Mato Grosso, Faculdade de Educação Física, Campus Cuiabá, Mato Grosso, Brasil

* Autor correspondente: Tenreiro Aranha, 2713, centro, Porto Velho - RO, CEP: 76801-114. E-mail: tatiane_edfisica@hotmail.com 


\section{INTRODUÇÃO}

A síndrome metabólica (SM) é uma combinação de fatores de risco cardiovasculares que inclui pressão arterial elevada, dislipidemia, resistência à insulina, hiperglicemia e obesidade abdominal (Tibana \& Prestes, 2013). Dada a crescente prevalência na população (Davila et al., 2013), a SM já é considerada uma epidemia mundial (Cameron, Shaw, \& Zimmet, 2004; Ford, Li, \& Zhao, 2010; Grundy, 2008), responsável por grande morbimortalidade tanto em países desenvolvidos como em desenvolvimento, o que implica, do ponto de vista populacional, em alto custo socioeconômico (Cameron et al., 2004; Nichols \& Moler, 2011).

Do ponto de vista individual, a SM representa não apenas um fator de risco para eventos cardiovasculares e metabólicos, mas trata-se de uma condição que tende a comprometer múltiplos aspectos da vida, incluindo a funcionalidade articular (Zhuo, Yang, Chen, \& Wang, 2012), a qualidade do sono (Ohkuma et al., 2014), a saúde bucal (Nibali et al., 2013), mental e psicológica (Nousen, Franco, \& Sullivan, 2013; Roohafza, Sadeghi, Talaei, Pourmoghaddas, \& Sarrafzadegan, 2012), podendo afetar, também, o convívio social (Sarrafzadegan et al., 2011), e a capacidade de trabalho (Schultz \& Edington, 2009).

Não obstante, recente revisão sistemática (de Carvalho Vidigal, Bressan, Babio, \& SalasSalvadó, 2013) demonstrou que na população brasileira a prevalência de SM tende a aumentar conforme a idade e a ser maior no sexo feminino. Dentre nove publicações incluídas no estudo em questão, cinco indicaram maior prevalência no sexo feminino e quatro não encontraram diferenças entre os sexos. Além disso, o estudo constatou também que a maior prevalência de SM se dá na faixa etária de 50 anos e mais. Sugere-se então que a fase da transição menopáusica possa ser um determinante no aumento da prevalência de SM, aumentando a preocupação com a prevenção em mulheres adultas (Kim, Park, Ryu, \& Kim, 2007).

Em relação à aptidão física, estudos anteriores demonstram que indivíduos com SM apresentam piores escores de aptidão cardiovascular (Laaksonen et al., 2002; LaMonte et al., 2005; Wijndaele et al., 2007), força muscular e força muscular relativa, definida como a força absoluta dividida pela massa corporal (Tibana et al., 2011; Tibana, Balsamo, \& Prestes, 2011), além de pior flexibilidade, em se tratando de mulheres idosas (Vieira et al., 2013). Estes comprometimentos na aptidão física tendem a afetar a mobilidade (Peterson, Snih, Stoddard, Shekar, \& Hurvitz, 2014), gerando dificuldade na realização de tarefas cotidianas de autocuidado e trabalho (Vieira et al., 2013), o que possivelmente compromete a qualidade de vida (QV) dos sujeitos com SM. Embora ainda não haja definição universalmente aceita de $\mathrm{QV}$, aquela proposta pelo WHOQOL Group foi adotada no presente estudo: a percepção do indivíduo sobre sua posição na vida, no contexto da cultura e do sistema de valores em que vive e em relação aos seus objetivos, expectativas, padróes e preocupaçôes (WHOQOL, 1995).

Vale ressaltar que múltiplos aspectos, além da capacidade física, interferem na classificação da QV. Trata-se de uma medida subjetiva, cujo conceito incorpora também o estado psicológico, nível de independência, as relações sociais, crenças pessoais e a relação com os aspectos significativos do meio ambiente (Azevedo, da Silva, Tomasi, \& Quevedo, 2013). Apesar de sua definição complexa, a QV é uma medida que tem importante correlação com a progressão das doenças crônicas e mortalidade (Schenkeveld et al., 2010; Yorgancioglu, Havlucu, Celik, Dinc, \& Saka, 2010).

Sendo assim, o objetivo primário do presente estudo foi comparar a QV e a aptidão física de mulheres com e sem SM. Outro objetivo foi examinar a correlação entre aptidão física (aptidão aeróbia e força muscular) e a QV nas mulheres com e sem SM. A hipótese inicial do presente estudo era de 
que as mulheres com SM teriam pior QV, aptidão aeróbia e força muscular. Além disso, hipotetizou-se que existiriam correlações entre a aptidão aeróbia, a força muscular e a QV.

\section{MÉTODO}

Foi realizado um estudo tranversal no período de agosto de 2011 a julho de 2013, com voluntárias recrutadas por anúncio público em jornais de circulação local e por contato telefônico a partir de cadastro existente na Universidade. As voluntárias foram selecionadas por conveniência e independentemente de etnia ou status socioeconômico. O critério de inclusão para as participantes foi: idade $\geq 18 \leq 50$ anos. Os critérios de exclusão foram: a) presença de doenças e/ou limitações cardiorrespiratórias, osteomioarticulares, cognitivas e/ou neurológicas que comprometessem a execução dos testes; b) ter realizado exercício físico sistematizado nos seis meses anteriores ao estudo; c) estar na menopausa por diagnóstico médico clínico (período de um ano ou mais sem menstruação) e exame endocrinológico complementar; e d) ser tabagista.

O estudo foi aprovado pelo Comitê de Ética e Pesquisa da Universidade Católica de Brasília (UCB). O termo de consentimento livre e esclarecido e um questionário de prontidão para a prática de atividade física (PAR-Q) foram obtidos de todas as voluntárias antes da inclusão na pesquisa. A amostra foi constituída por 49 voluntárias, organizadas em dois grupos: 21 mulheres com SM (33.5 \pm 9.1 anos; $\left.32.6 \pm 3.7 \mathrm{~kg} / \mathrm{m}^{2}\right)$ e $28 \mathrm{sem} \mathrm{SM}(32.3 \pm 7.9$ anos; $27.1 \pm 3.2 \mathrm{~kg} / \mathrm{m}^{2}$ ).

\section{Síndrome metabólica}

A organização dos grupos foi feita a partir dos critérios propostos pela National Cholesterol Education Program - Adult Treatment Panel III (NCEP-ATP III), sendo incluídas no grupo $\mathrm{SM}$ as mulheres que apresentavam três ou mais dos seguintes componentes: obesidade central (circunferência abdominal $>88 \mathrm{~cm}$ ), hipertrigliceridemia (TG $>150 \mathrm{mg} / \mathrm{dl}$ ), baixo HDL-c $(<50 \mathrm{mg} / \mathrm{dL})$, pressão arterial elevada (pressão arterial sistólica [PAS] $>130 \mathrm{mmHg}$ e/ou pressão arterial diastólica [PAD] $>85$ mmHg e/ou tratamento para hipertensão arterial), hiperglicemia (glicemia de jejum > $100 \mathrm{mg} / \mathrm{dL}$ e/ou tratamento para o diabetes tipo II).

\section{Pressão arterial}

A determinação da pressão arterial sistólica (PAS) e diastólica (PAD) foi realizada de acordo com a metodologia proposta pela $\mathrm{V}$ Diretriz Brasileira de Hipertensão Arterial (Sociedade Brasileira de Cardiologia, 2010). Os procedimentos de medida foram realizados em uma sala com ambiente controlado $\left(\sim 22^{\circ} \mathrm{C}\right)$, sendo utilizado como instrumento um medidor oscilométrico (Microlife 3AC1- 1, Widnau, Suíça). Previamente à medição a voluntária permaneceu sentada, em repouso, durante 10 minutos. Em seguida o braço esquerdo foi apoiado para permanecer em posição relaxada e, ao nível do coração, foi colocada a braçadeira do aparelho três centrímetros acima da fossa antecubital, centralizando o manguito sobre a artéria umeral. Foram realizadas três medidas, com intervalo de um minuto, sendo utilizada a média das mesmas.

\section{Análises bioquímicas}

A coleta de sangue foi realizada mediante punção de sangue venoso na veia antecubital, após jejum de 12 horas. As concentrações de triglicerídeos, lipoproteína de alta densidade (HDL-C) e colesterol total foram determinadas pelo método enzimático automatizado (Autohumalyzer,Human GMBH, Alemanha) e a glicemia pelo método enzimático por hexoquinase (Cobas Mira Plus, Roche, Minnesota, USA) realizadas no laboratório de análises clínicas Universidade Católica de Brasília.

\section{Antropometria e composição corporal}

A medida da massa corporal foi realizada com a voluntária descalça, vestindo roupas 
leves, e utilizando-se balança digital (WelmyW110H, São Paulo, Brasil) com capacidade de $150 \mathrm{~kg}$ e divisão de $100 \mathrm{~g}$. A estatura foi medida por um estadiômetro de parede (Sanny, São Paulo, Brasil), com capacidade de $2200 \mathrm{~mm}$ e divisão de $1 \mathrm{~mm}$. As circunferências (cintura, pescoço e quadril) foram medidas com uma fita métrica (Sanny, Brasil), e durante a medição as participantes permaneceram de pé, na posição ereta, com a cabeça posicionada no plano horizontal de Frankfurt. A circunferência da cintura foi medida na distância média entre a última costela flutuante e a crista ilíaca. Para a medida da circunferência do pescoço, a borda superior da fita métrica foi colocada logo abaixo da proeminência da laringe e aplicada perpendicularmente ao longo do eixo do pescoço (Tibana et al., 2012). Os valores do índice de adiposidade corporal (IAC) foram calculados por meio da fórmula:

\section{IAC $=[($ circunferência do quadril/estatura $) \times 1.5)-18)]$}

A circunferência do quadril foi medida ao nível dos grandes trocânters femorais, no local de maior circunferência. Todas as medidas foram tomadas por um avaliador experiente e com três medidas em cada posição.

A composição corporal foi determinada por meio da técnica de espessura das dobras cutâneas, realizada sempre pelo mesmo avaliador, sendo utilizado como instrumento um compasso Lange, e como pontos anatômicos as regiões: peitoral, abdominal e coxa, de acordo com o protocolo de Jackson, Pollock, e Ward (1980). A confiabilidade foi de $R=0.83(0.62-0.92)$.

\section{Qualidade de vida}

A QV foi avaliada pelo questionário de saúde Short Form Health Survey 36 (SF-36), que contém 36 itens agrupados em oito domínios: capacidade funcional física, limitação por aspectos físicos, dor corporal, estado de saúde geral, vitalidade, aspectos sociais da funcionalidade, aspectos emocionais e saúde mental. A variação da pontuação é de 0 a 100 em cada domínio, ao passo que uma maior pontuação indica melhores condições de saúde relacionadas à QV (Ciconelli, Ferraz, Santos, Meinão, \& Quaresma, 1999). O estudo de validação do SF-36 de Ciconelli, Ferraz, Santos, Meinão, e Quaresma (1999) demonstrou uma reprodutibilidade intra observadores para a capacidade funcional de 0.80 , aspectos físicos 0.63 , dor 0.54 , estado geral de saúde 0.84 , vitalidade 0.65 , aspectos sociais 0.75 , aspectos emocinais 0.44 e saúde mental 0.69. O referido instrumento foi preenchido por entrevista, realizada sempre pelo mesmo avaliador.

\section{Aptidão aeróbia: Teste de caminhada de 6 minutos}

O teste de 6 minutos de caminhada seguiu o protocolo do ATS (American Thoracic Society). A capacidade aeróbia foi determinada pela distância percorrida em um corredor coberto, de 30 metros de comprimento e livre da circulação de pessoas. O teste foi realizado pelo menos duas horas após a última refeição. As voluntárias foram instruídas a usar roupas e calçados confortáveis (Brooks, Solway, \& Gibbons, 2003).

\section{Força de Preensão Manual}

A força de preensão manual foi obtida com dinamômetro mecânico manual (Takei, T. K. K Grip strength dinamometer 0-100 kg, Japão). As mulheres permaneceram em pé com os dois braços estendidos e antebraço em rotação neutra. Para todos os sujeitos a pegada do dinamômetro foi ajustada individualmente, de acordo com o tamanho das mãos, de forma que a haste mais próxima do corpo do dinamômetro estivesse posicionada sobre as segundas falanges dos dedos indicador, médio e anular. O período de recuperação entre as medidas foi de cerca de um minuto e o teste foi realizado em três tentativas em ambas as mãos. A melhor marca das três tentativas foi utilizada como medida (Farias, Teixeira, Tibana, Balsamo, \& Prestes, 2012). A força muscular 
relativa foi calculada por meio da seguinte equação (Prestes \& Tibana, 2013):

Força relativa $=$ Força absoluta $(\mathrm{kg}) /$ Massa corporal $(\mathrm{kg})$

\section{Análise estatística}

Inicialmente, foi realizada a análise descritiva da amostra com medidas de tendência central e dispersão. A normalidade dos dados foi confirmada a partir do teste de Kolmogorov-Smirnov. Para a comparação dos grupos foi utilizado o teste $\mathrm{t}$ de Student não pareado (todos os dados foram paramétricos). A correlação entre os parâmetros da aptidão física (força muscular relativa e teste de 6 minutos de caminhada) e os parâmetros de qualidade de vida (SF-36) foi testada por meio da correlação de Pearson. O nível de significância para todas as variáveis estudadas foi de $\mathrm{p} \leq 0,05$. Estimou-se uma amostra mínima de 20 voluntárias para cada grupo, com um poder de teste (power) de $90 \%$ para indicar diferenças entre os grupos, sendo o tamanho do efeito de .97. Para o cálculo do tamanho do efeito o g de Hedges foi utilizado e valores entre $0,20-0,50,0,50-0,80$ e maior que 0,80 foram considerados pequeno, médio e grande, respectivamente. Os dados foram analisados por meio do programa GraphPad Prism ${ }^{\circledR}$, versão 6.0, San Diego, CA, Estados Unidos.

\section{RESULTADOS}

Os parâmetros antropométricos, pressóricos e bioquímicos das mulheres com e sem SM estão apresentados nas tabelas 1 e 2 . As mulheres com SM apresentaram maiores valores de massa corporal, IMC, circunferência da cintura, circunferência do quadril, circunferência do pescoço, razão cinturaestatura, razão cintura-quadril, índice de adiposidade corporal, percentual de gordura, massa gorda, massa livre de gordura, PAS, PAD, glicemia, triglicerídeos, LDL-C, colesterol total e razão colesterol total/HDL quando comparadas às mulheres sem SM. Por outro lado, as mulheres com SM exibiram menores valores de HDL-C, força muscular relativa e distância percorrida no teste de 6 minutos (figura 1). No entanto, para a força muscular absoluta o grupo com SM apresentou maior força muscular quando comparadas com as mulheres sem SM, mas sem diferença estatística entre os grupos.

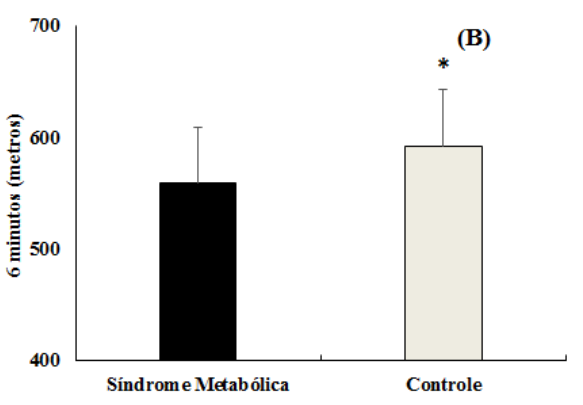

(C)

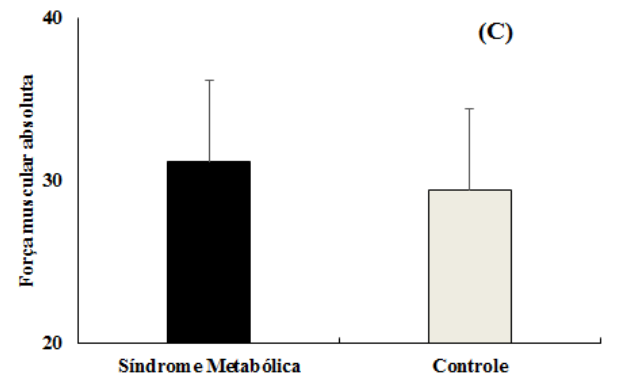

Figura 1. Força muscular relativa (A), distância percorrida no teste de 6 minutos de caminhada (B) e força muscular absoluta (C) de mulheres com e sem síndrome metabólica. *Diferença significativa entre os grupos. 
Tabela 1

Características antropométricas.

\begin{tabular}{|c|c|c|c|c|c|c|c|}
\hline Variáveis & $\begin{array}{c}\text { Síndrome } \\
\text { Metabólica } \\
(\mathrm{n}=21)\end{array}$ & $\begin{array}{l}\text { Valor mínimo e } \\
\text { máximo }\end{array}$ & $\begin{array}{l}\text { Controle } \\
(n=28)\end{array}$ & $\begin{array}{l}\text { Valor mínimo e } \\
\text { máximo }\end{array}$ & $\begin{array}{l}\text { Diferença entre as } \\
\text { médias }(95 \%-I C)\end{array}$ & $p$ & $\mathrm{TE}$ \\
\hline Idade (anos) & $33.5 \pm 9.1$ & $20.00-49.00$ & $32.3 \pm 7.9$ & $18.00-50.00$ & $1.16(-3.7 / 6.0)$ & .63 & 0.14 \\
\hline Estatura (m) & $1.60 \pm .08$ & $1.44-1.75$ & $1.60 \pm .04$ & $1.50-1.66$ & $0.0(-0.03 / .03)$ & 1.00 & 0.05 \\
\hline Massa Corporal (kg) & $83.9 \pm 15.6$ & $60.00-104.40$ & $69.5 \pm 9.5^{*}$ & $49.9-89.10$ & $14.4(7.2 / 21.6)$ & .001 & 1.52 \\
\hline $\operatorname{IMC}\left(\mathrm{kg} / \mathrm{m}^{2}\right)$ & $32.6 \pm 3.7$ & $27.36-39.57$ & $27.1 \pm 3.2^{*}$ & $20.87-33.12$ & $5.5(3.5 / 7.5)$ & .001 & 1.74 \\
\hline C. da cintura (cm) & $92.5 \pm 8.3$ & $83.0-109.00$ & $81.5 \pm 6.9^{*}$ & $65.00-95.00$ & $11.0(6.6 / 15.3)$ & .001 & 1.59 \\
\hline C. do Quadril (cm) & $111.6 \pm 10.1$ & $94.00-132.00$ & $103.7 \pm 6.4^{*}$ & $89.00-118.00$ & $7.9(3.1 / 12.6)$ & .001 & 1.24 \\
\hline C. do Pescoço $(\mathrm{cm})$ & $36.6 \pm 2.3$ & $32.00-41.00$ & $34.1 \pm 2.3^{*}$ & $31.00-39.00$ & $2.5(1.2 / 3.8)$ & .001 & 1.09 \\
\hline Razão Cintura/Estatura & $0.58 \pm .06$ & $0.49-0.75$ & $0.51 \pm .04^{*}$ & $0.40-0.59$ & $0.07(.04 / .09)$ & .001 & 1.64 \\
\hline Razão Cintura/Quadril & $0.83 \pm .08$ & $0.69-1.05$ & $0.79 \pm .05^{*}$ & $0.68-0.92$ & $0.04(.002 / .07)$ & .03 & 0.93 \\
\hline IAC (\%) & $28.5 \pm 4.6$ & $19.01-37.52$ & $25.2 \pm 2.5^{*}$ & $18.77-31.47$ & $3.3(1.2 / 5.4)$ & .002 & 1.31 \\
\hline$\%$ de Gordura & $39.4 \pm 4.3$ & $31.97-45.47$ & $35.1 \pm 5.5^{*}$ & $22.80-44.72$ & $4.3(1.4 / 7.2)$ & .004 & 0.77 \\
\hline MLG (kg) & $51.1 \pm 6.4$ & $38.63-61.36$ & $44.5 \pm 4.1^{*}$ & $36.95-51.49$ & $6.6(3.5 / 9.6)$ & .001 & 1.60 \\
\hline Massa Gorda (kg) & $33.6 \pm 7.4$ & $21.37-45.30$ & $24.7 \pm 6.6^{*}$ & $12.33-37.61$ & $8.9(4.8 / 12.9)$ & .001 & 1.34 \\
\hline
\end{tabular}

C. = Circunferência; IMC = Índice de massa corporal; IAC = Índice de adiposidade corporal; IC = intervalo de confiança; MLG = Massa livre de gordura ${ }^{*}$ Diferença significativa entre os grupos, TE = tamanho do efeito. 
54 | TG Teixeira, RA Tibana, DC Nascimento, RAS Silva, JA Almeida, S Balsamo, FA Voltarelli, J Prestes

Tabela 2

Resultados de pressão arterial, glicemia e perfil lipídico de mulheres com Síndrome Metabólica e controle

\begin{tabular}{|c|c|c|c|c|c|c|c|}
\hline Variáveis & $\begin{array}{c}\text { Síndrome Meta- } \\
\text { bólica } \\
(n=21)\end{array}$ & $\begin{array}{l}\text { Valor mínimo e } \\
\text { valor máximo }\end{array}$ & $\begin{array}{l}\text { Controle } \\
(n=28)\end{array}$ & $\begin{array}{l}\text { Valor mínimo e } \\
\text { valor máximo }\end{array}$ & $\begin{array}{l}\text { Diferença entre as } \\
\text { médias }(95 \% \text { - IC) }\end{array}$ & $P$ & TE \\
\hline PAS (mmHg) & $130.3 \pm 15.4$ & $97.00-164.00$ & $113.8 \pm 8.9^{*}$ & $99.00-142.00$ & $16.5(9.4 / 23.5)$ & .001 & 1.83 \\
\hline PAD (mmHg) & $84.6 \pm 15.3$ & $53.00-113.00$ & $75.6 \pm 7.7^{*}$ & $59.00-89.00$ & $9.0(2.3 / 15.7)$ & .009 & 1.18 \\
\hline Glicemia (mg/dL) & $99.3 \pm 27.1$ & $80.00-207.00$ & $85.3 \pm 7.5^{*}$ & 67.0098 .00 & $14.0(3.2 / 24.8)$ & .01 & 1.89 \\
\hline Triglicerídeos (mg/dL) & $140.2 \pm 61.9$ & $49.00-250.00$ & $84.8 \pm 31.0^{*}$ & $30.00-146.00$ & $55.4(28.3 / 82.5)$ & .001 & 1.79 \\
\hline HDL-C (mg/dL) & $43.4 \pm 11.4$ & $27.00-66.00$ & $50.7 \pm 12.5^{*}$ & $25.00-81.00$ & $-7.3(-14.3 /-0.3)$ & .04 & 0.58 \\
\hline LDL-C (mg/dL) & $127.9 \pm 33.0$ & & $101.3 \pm 26.8^{*}$ & & $26.6(9.4 / 43.75)$ & .003 & 1.06 \\
\hline Colesterol (mg/dL) & $210.1 \pm 36.4$ & $171.00-307.00$ & $176.3 \pm 39.1^{*}$ & $124.00-276.00$ & $33.8(11.7 / 55.8)$ & .001 & 0.86 \\
\hline Colesterol/HDL-C & $4.5 \pm 0.8$ & $2.64-5.58$ & $3.7 \pm 1.2^{*}$ & $2.10-7.16$ & $0.8(0.19 / 1.4)$ & .01 & 0.66 \\
\hline
\end{tabular}

PAS = Pressão arterial sistólica; PAD = Pressão arterial diastólica; IC = Intervalo de confiança; LDL = Low Density Lipoprotein; HDL = High Densitiy Lipoprotein. *Diferença significativa entre os grupos, TE = tamanho do efeito. 
Os dados referentes à QV estão apresentados na Tabela 3. O SF-36 apontou que as mulheres com SM tiveram pior QV nos domínios: estado de saúde geral, capacidade funcional, dor no corpo, limitação por aspectos emocionais, aspectos sociais da funcionalidade e limitação por aspectos físicos, quando comparadas às mulheres sem SM.

Tabela 3

Scores de qualidade de vida (SF-36) de mulheres com e sem síndrome metabólica.

\begin{tabular}{lccccc}
\hline Variáveis & $\begin{array}{c}\text { Síndrome } \\
\text { Metabólica } \\
(\mathbf{n}=\mathbf{2 1})\end{array}$ & $\begin{array}{c}\text { Controle } \\
(\mathbf{n}=\mathbf{2 8})\end{array}$ & $\begin{array}{c}\text { Diferença entre as } \\
\text { médias }(95 \%-\mathrm{IC})\end{array}$ & $p$ & TE \\
\hline Capacidade funcional & $75.2 \pm 15.4$ & $85.4 \pm 13.7^{*}$ & $-10.2(-18.6 /-1.8)$ & .01 & 0.74 \\
Limitação A. Físicos & $66.7 \pm 26.6$ & $93.7 \pm 12.9^{*}$ & $-27.0(-38.6 /-15.4)$ & .001 & 2.09 \\
Dor no corpo & $60.1 \pm 23.7$ & $74.6 \pm 20.2^{*}$ & $-14.5(-27.1 /-1.8)$ & .02 & 0.72 \\
Estado de saúde geral & $58.7 \pm 21.8$ & $73.4 \pm 18.1^{*}$ & $-14.7(-26.2 /-3.2)$ & .01 & 0.82 \\
Vitalidade & $56.7 \pm 11.2$ & $53.9 \pm 9.2$ & $2.8(-3.1 / 8.6)$ & .34 & 0.30 \\
A. S. funcionalidade & $78.1 \pm 19.4$ & $87.9 \pm 12.0^{*}$ & $-9.8(-18.5 /-0.7)$ & .03 & 0.81 \\
Limitação A. emocionais & $66.6 \pm 34.9$ & $86.4 \pm 23.1^{*}$ & $-19.8(-36.4 /-3.1)$ & .02 & 0.87 \\
Saúde mental & $60.0 \pm 9.7$ & $59.1 \pm 9.2$ & $0.9(-4.5 / 6.3)$ & .74 & 0.09 \\
\hline
\end{tabular}

A. $=$ por aspectos; A.S. $=$ aspectos sociais da; IC $=$ intervalo de confiança. ${ }^{*}$ Diferençasignificativa entre os grupos. $\mathrm{TE}=$ tamanho do efeito.

Na tabela 4 estão apresentadas todas as correlações estatisticamente significantes entre o teste de caminhada de 6 minutos e os domínios da QV (SF-36). Foram observadas correlações positivas com os domínios: capacidade funcional, limitação por aspecto físico, dor e aspectos sociais. Não foram observadas correlações entre a força muscular relativa e os parâmetros de $\mathrm{QV}$ (dados não demonstrados graficamente).

Tabela 4

Correlação do teste de 6 minutos de caminhada com capacidade funcional, limitação por aspetos físicos, dor e aspetos sociais.

\begin{tabular}{lc}
\hline \multicolumn{1}{c}{ Domínios do SF-36 } & $\mathbf{r}(95 \%-\mathrm{IC})$ \\
\hline Capacidade Funcional & $0.30^{*}(0.02-0.52)$ \\
Limitação por aspectos & $0.33^{*}(0.05-0.54)$ \\
físicos & $0.25^{*}(-0.03-0.49)$ \\
Dor & $0.34^{*}(0.06-0.55)$ \\
Aspectos sociais & \\
\hline IC $=$ intervalo de confiança, $\quad(\mathrm{n}=49) \quad\left({ }^{*} \mathrm{p}<.01 ;\right.$ \\
$* * \mathrm{p}<.05)$.
\end{tabular}

\section{DISCUSSÃO}

Os dados dos testes confirmam nossa hipótese, já que as mulheres com SM apresentaram menor aptidão aeróbia, força muscular e pior QV quando comparadas ao grupo-controle. Adicionalmente, foi observada correlação entre a aptidão aeróbia e quatro domínios da QV: capacidade funcional, limitação por aspectos físicos, dor no corpo e aspectos sociais. Os resultados do presente estudo confirmam as consequências negativas da SM sobre a $\mathrm{QV}$, força muscular e aptidão aeróbia, o que aumenta a preocupação com saúde de mulheres com SM.

Wijndaele et al., (2007) analisaram a associação entre a força muscular e aptidão aeróbia com os fatores de risco da SM em 1019 homens e mulheres (18-75 anos). A força muscular foi avaliada pelo teste de força isométrica de extensão do joelho e o torque da flexão do joelho. Já aptidão aeróbia foi determinada por meio de um teste máximo em cicloergômetro. Os resultados encontrados pelos pesquisadores demonstraram que a força muscular foi inversamente associada com a SM ( $\beta$ $=-0.17 ; \mathrm{P}<0.001)$ apenas nas mulheres e a aptidão aeróbia foi inversamente associada com a SM tanto em homens $(\beta=-0.31 ; \mathrm{P}<$ $0.001)$ como nas mulheres $(B=-0.21, P<$ $0.001)$. 
Tibana, Tajra, et al., (2011) compararam a força muscular de preensão manual em mulheres brasileiras com e sem SM. Os resultados encontrados pelos autores foram similares ao do presente estudo, no qual mulheres com SM apresentam menor força muscular relativa quando comparado às mulheres sem SM.

Farias et al. (2013) analisaram a força muscular (10 repetições máximas na cadeira extensora) de idosas com e sem SM. Os autores demonstraram que, além de possuírem piores escores de risco cardiovascular, as mulheres com SM apresentavam menor força muscular relativa quando comparadas às idosas sem SM.

Mais recentemente, Vieira et al. (2013) compararam os fatores de risco cardiovascular, a flexibilidade, a força muscular, a capacidade funcional e aptidão aeróbia (6 minutos de caminhada) de idosas com e sem SM. Semelhante aos estudos anteriores, os autores demonstraram que idosas com SM apresentaram pior saúde cardiovascular, menor força muscular relativa do leg press (1.44 \pm .40 versus $1.78 \pm .55)$ e menor aptidão cardiovascular $(458.4 \pm 80.1$ versus $527.9 \pm$ 33.9) do que as idosas sem SM. Os autores ainda encontraram correlação entre a aptidão cardiovascular e o número de fatores de risco da SM ( $r=-.44 ; \quad p=.01)$. Resultados semelhantes foram encontrados no presente estudo, no qual as mulheres com SM percorreram menor distância no teste de caminhada de 6 minutos e tiveram menor força muscular relativa quando comparado às mulheres sem SM.

Dentre as alterações fisiopatológicas que podem justificar a pior condição física das mulheres com SM destacamos: a) a menor capacidade miocárdica (Li et al., 2012), b) a condição endotelial deficiente, que resulta tanto das alterações lipídicas e pressóricas quanto da síntese de adipocinas pelo tecido adiposo (Van Gaal, Mertens, \& De Block, 2006; Cornier et al., 2008), e gera menor capacidade de vasodilatação (Kotsis, Stabouli,
Papakatsika, Rizos, \& Parati, 2010); e c) a menor capacidade muscular de captar glicose, como consequência da resistência à ação da insulina (Holloszy, 2005; Jessen \& Goodyear, 2005).

Possivelmente os mecanismos em questão explicam os resultados do presente estudo e também de publicações anteriores que demonstram piores condições de aptidão física em sujeitos com SM. Por outro lado, o sedentarismo elevado na população com SM (Laaksonen et al., 2002), pode ser um importante influenciador destes resultados. $\mathrm{O}$ excesso de adiposidade, presente na maior parte dos sujeitos com SM, pode gerar maior fadiga e desconforto articular durante a realização de esforços físicos, tornando o sujeito menos propenso à prática regular de atividades físicas, culminando em descondicionamento.

Possivelmente o conjunto de alterações fisiopatológicas afeta a capacidade de realizar atividades da vida pessoal e profissional, e geram no sujeito menor percepção de saúde e capacidade física, em comparação aos sujeitos sem as alterações patológicas da SM. Estes aspectos se fizeram perceber através da avaliação auto-percebida de qualidade de vida, realizada neste e em estudos anteriores.

Sarrafzadegan et al. (2011) analisaram a associação entre SM e qualidade de vida em 9570 Iranianos (em torno de 38 anos). A prevalência de SM na amostra estudada foi de $22,5 \%$, e apenas as mulheres sem SM tiveram melhores scores de saúde física e relações sociais quando comparado às mulheres com $\mathrm{SM}$, ao passo que não foram observadas diferenças significativas entre os homens com e sem SM. Este resultado indica que o impacto da SM é diferente em homens e mulheres,

Outro resultado interessante do presente estudo foi a correlação entre a aptidão aeróbia e a QV nas mulheres com e sem SM, reforçando que as adaptações fisiopatológicas da SM, descritas em estudos prévios, implicam não apenas em risco cardiovascular aumentado, mas também apresentam 
consequências diretas sobre a vida cotidiana de indivíduos com SM. Ademais, foi observada correlação entre a aptidão aeróbia e dois parâmetros da QV: limitação por aspectos físicos e dor no corpo. Este resultado fortalece a hipótese de que a maior fadiga e dores articulares nos sujeitos com SM são limitantes indiretos da aptidão física, como consequência de menor capacidade de realização de exercícios físicos.

Estes resultados indicam que testes relativamente simples podem colaborar para avaliar a condição funcional de indivíduos com SM; e sugerem que a modificação do estilo de vida, especialmente da aptidão aeróbia e força muscular são importantes medidas para a prevenção e o tratamento da SM.

De fato, estudos recentes demonstram que programas de exercícios físicos beneficiam a aptidão física e a qualidade de vida de indivíduos com SM. Eleutério-Silva et al., (2013) demonstraram que um programa de exercício de 18 sessões (6 semanas), composto de exercícios aeróbios e resistidos, foi suficiente para melhorar a aptidão aeróbia (teste de 6 minutos), a função vascular, a pressão arterial e o sistema de defesa antioxidante de mulheres com idade entre $30 \mathrm{e}$ 60 anos, portadoras de SM.

Os resultados dos estudos anteriores, juntamente com a correlação entre a aptidão aeróbia e a QV observada no presente estudo sugerem que a aptidão física é um importante componente da percepção de qualidade de vida dos indivíduos com SM. Por consequência, a inclusão de exercícios físicos é uma estratégia potencialmente eficaz para minimizar as consequências negativas associadas à SM. Vale ressaltar que algumas comparações entre os resultados do presente estudo com os de outros trabalhos ficam limitadas devido as diferentes populações, faixa etária e testes utilizados.

Foram limitações do presente estudo o delineamento transversal, que não permite interpretação de causa e efeito, a ausência de medidas de condição econômica e estado civil e também a ausência de medidas de reprodutibilidade no questionário SF-36. O uso de testes indiretos, ao invés de protocolos laboratoriais, para a avaliação da aptidão aeróbia (teste de caminhada de 6 minutos) e força muscular (teste de prensão manual ou handrip) também podem ser apontados como limitações. Entretanto ambos tem sido largamente utilizados na literatura, em razão de possuírem metodologia de fácil aplicabilidade e de não necessitar de equipamentos sofisticados e de grande porte, bem como de pessoal altamente treinado, razão pela qual sua validade ecológica deve ser considerada. Além disso, o teste de caminhada de 6 minutos tem se mostrado muito útil para diagnóstico de capacidade funcional, estado clínico e prognóstico cardiovascular em portadores de doenças crônicas, (Golpe et al., 2014; Golpe, Pérez-de-Llano, Méndez-Marote, \& Veres-Racamonde, 2013), sendo bem aceito e seguro em pacientes com insuficiência cardíaca crônica e doença pulmonar crônica (Rubim, Drumond Neto, Romeo, \& Montera, 2006); enquanto o handgrip apresenta boa correlação com medidas diretas da força, como o teste de extensão de joelhos (Bohannon, Magasi, Bubela, Wang, \& Gershon, 2012).

\section{CONCLUSÕES}

Os resultados deste estudo demonstraram que, em comparação às mulheres sem SM, mulheres com SM apresentam menor força muscular e aptidão aeróbia, bem como piores escores em seis domínios de QV (estado de saúde geral, capacidade funcional, dor no corpo, limitação por aspectos emocionais, aspectos sociais da funcionalidade e limitação por aspectos físicos). Além disso, foi constatada correlação significativa entre quatro domínios da qualidade de vida (capacidade funcional, limitação por aspecto físico, dor e aspectos sociais da funcionalidade) e a aptidão aeróbia de mulheres com e sem SM. 


\section{Agradecimentos:}

Nada a declarar

\section{Conflito de Interesses:}

Nada a declarar.

\section{Financiamento:}

Nada a declarar.

\section{REFERÊNCIAS}

Azevedo, A. L. S. de, da Silva, R. A., Tomasi, E., \& Quevedo, L. de Á. (2013). Chronic diseases and quality of life in primary health care. $\mathrm{Ca}$ dernos de Saúde Pública, 29(9), 1774-1782. http://doi.org/10.1590/0102-311X00134812

Bohannon, R. W., Magasi, S. R., Bubela, D. J., Wang, Y.-C., \& Gershon, R. C. (2012). Grip and Knee extension muscle strength reflect a common construct among adults. Muscle \& Nerve, 46(4), 555-558. http://doi.org/10.1002/mus.23350

Brooks, D., Solway, S., \& Gibbons, W. J. (2003). ATS statement on six-minute walk test. American Journal of Respiratory and Critical Care Medicine, $167(9), \quad 1287$. http://doi.org/10.1164/ajrccm.167.9.950

Cameron, A. J., Shaw, J. E., \& Zimmet, P. Z. (2004). The metabolic syndrome: prevalence in worldwide populations. Endocrinology and Metabolism Clinics of North America, 33(2), 351-375. http://doi.org/10.1016/j.ecl.2004.03.005

Ciconelli, R. M., Ferraz, M. B., Santos, W., Meinão, I., \& Quaresma, M. R. (1999). Tradução para a língua portuguesa e validação do questionário genérico de avaliação de qualidade de vida SF36 (Brasil SF-36). Revista Brasileira de Reumatologia, 39(3), 143-50.

Cornier, M.-A., Dabelea, D., Hernandez, T. L., Lindstrom, R. C., Steig, A. J., Stob, N. R., ... Eckel, R. H. (2008). The Metabolic Syndrome. Endocrine Reviews, 29(7), 777-822. http://doi.org/10.1210/er.2008-0024

Davila, E. P., Quintero, M. A., Orrego, M. L., Ford, E. S., Walke, H., Arenas, M. M., \& Pratt, M. (2013). Prevalence and risk factors for metabolic syndrome in Medellin and surrounding municipalities, Colombia, 2008-2010. Preventive $\quad$ Medicine, 56(1), 30-34. http://doi.org/10.1016/j.ypmed.2012.10.027 de Carvalho Vidigal, F., Bressan, J., Babio, N., \& Salas-Salvadó, J. (2013). Prevalence of metabolic syndrome in Brazilian adults: a systematic review. BMC Public Health, 13(1), 1198. http://doi.org/10.1186/1471-2458-13-1198

Eleutério-Silva, M. A., Sá da Fonseca, L. J., Velloso, E. P. P., da Silva Guedes, G., Sampaio, W. O., da Silva, W. F., ... Rabelo, L. A. (2013). Shortterm cardiovascular physical programme ameliorates arterial stiffness and decreases oxidative stress in women with metabolic syndrome. Journal of Rehabilitation Medicine, 45(6), 572-579. http://doi.org/10.2340/165019771148

Farias, D. L., Teixeira, T. G., Tibana, R. A., Balsamo, S., \& Prestes, J. (2012). A força de preensão manual é preditora do desempenho da força muscular de membros superiores e inferiores em mulheres sedentárias. Motricidade, 8(Supl. 2), 624-629.

Farias, D. L., Tibana, R. A., Teixeira, T. G., Vieira, D. C. L., Tarja, V., Nascimento, D. da C., ... Prestes, J. (2013). Elderly women with metabolic syndrome present higher cardiovascular risk and lower relative muscle strength. Einstein (São Paulo), 11(2), 174-179. http://doi.org/10.1590/S167945082013000200007

Ford, E. S., Li, C., \& Zhao, G. (2010). Prevalence and correlates of metabolic syndrome based on a harmonious definition among adults in the US. Journal of Diabetes, 2(3), 180-193. http://doi.org/10.1111/j.17530407.2010.00078.x

Golpe, R., Castro-Añón, O., Pérez-de-Llano, L. A., González-Juanatey, C., Muñiz-Fernández, C., Testa-Fernández, A., \& Pérez-Fernández, R. (2014). Prognostic significance of six-minute walk test in non-group 1 pulmonary hypertension. Heart \& Lung: The Journal of Critical Care, 43(1), 72-76. http://doi.org/10.1016/j.hrtlng.2013.08.007

Golpe, R., Pérez-de-Llano, L. A., Méndez-Marote, L., \& Veres-Racamonde, A. (2013). Prognostic value of walk distance, work, oxygen saturation, and dyspnea during 6-minute walk test in COPD patients. Respiratory Care, 58(8), 1329-1334. http://doi.org/10.4187/respcare.02290

Grundy, S. M. (2008). Metabolic syndrome pandemic. Arteriosclerosis, Thrombosis, and Vascular Biology, 28(4), 629-636. http://doi.org/10.1161/ATVBAHA.107.15109 2

Holloszy, J. O. (2005). Exercise-induced increase in muscle insulin sensitivity. Journal of Applied Physiology (Bethesda, Md.: 1985), 99(1), 338343.

http://doi.org/10.1152/japplphysiol.00123.20 05 
Jackson, A. S., Pollock, M. L., \& Ward, A. (1980). Generalized equations for predicting body density of women. Medicine and Science in Sports and Exercise, 12(3), 175-181. http://doi.org/10.1249/00005768-19802300000009

Jessen, N., \& Goodyear, L. J. (2005). Contraction signaling to glucose transport in skeletal muscle. Journal of Applied Physiology (Bethesda, Md.: 1985), 99(1), 330-337. http://doi.org/10.1152/japplphysiol.00175.20 05

Kim, H. M., Park, J., Ryu, S. Y., \& Kim, J. (2007). The effect of menopause on the metabolic syndrome among Korean women: the Korean National Health and Nutrition Examination Survey, 2001. Diabetes Care, 30(3), 701-706. http://doi.org/10.2337/dc06-1400

Kotsis, V., Stabouli, S., Papakatsika, S., Rizos, Z., \& Parati, G. (2010). Mechanisms of obesityinduced hypertension. Hypertension Research: Official Journal of the Japanese Society of $\mathrm{Hy}_{\mathrm{y}}$ pertension, 33(5), 386-393. http://doi.org/10.1038/hr.2010.9

Laaksonen, D. E., Lakka, H.-M., Salonen, J. T., Niskanen, L. K., Rauramaa, R., \& Lakka, T. A. (2002). Low levels of leisure-time physical activity and cardiorespiratory fitness predict development of the metabolic syndrome. Diabetes Care, 25(9), 1612-1618.

LaMonte, M. J., Barlow, C. E., Jurca, R., Kampert, J. B., Church, T. S., \& Blair, S. N. (2005). Cardiorespiratory fitness is inversely associated with the incidence of metabolic syndrome: a prospective study of men and women. Circulation, 112(4),

505-512. http://doi.org/10.1161/CIRCULATIONAHA.1 04.503805

Li, Z.-L., Woollard, J. R., Ebrahimi, B., Crane, J. A., Jordan, K. L., Lerman, A., ... Lerman, L. O. (2012). Transition from obesity to metabolic syndrome is associated with altered myocardial autophagy and apoptosis. Arteriosclerosis, Thrombosis, and Vascular Biology, 32(5), 1132-1141.

http://doi.org/10.1161/ATVBAHA.111.24406 1

Nibali, L., Tatarakis, N., Needleman, I., Tu, Y.-K., D'Aiuto, F., Rizzo, M., \& Donos, N. (2013). Clinical review: Association between metabolic syndrome and periodontitis: a systematic review and meta-analysis. The Journal of Clinical Endocrinology and Metabolism, 98(3), 913920. http://doi.org/10.1210/jc.2012-3552

Nichols, G. A., \& Moler, E. J. (2011). Metabolic syndrome components are associated with future medical costs independent of cardiovascular hospitalization and incident diabetes. Metabolic Syndrome and Related Disorders, 9(2),
127-133.

http://doi.org/10.1089/met.2010.0105

Nousen, E. K., Franco, J. G., \& Sullivan, E. L. (2013). Unraveling the mechanisms responsible for the comorbidity between metabolic syndrome and mental health disorders. Neuroendocrinology, 98(4), 254-266. http://doi.org/10.1159/000355632

Ohkuma, T., Fujii, H., Iwase, M., Ogata-Kaizu, S., Ide, H., Kikuchi, Y., ... Kitazono, T. (2014). Ushaped association of sleep duration with metabolic syndrome and insulin resistance in patients with type 2 diabetes: the Fukuoka Diabetes Registry. Metabolism: Clinical and $E_{X-}$ perimental, 63(4), 484-491. http://doi.org/10.1016/j.metabol.2013.12.001

Peterson, M. D., Snih, S. Al, Stoddard, J., Shekar, A., \& Hurvitz, E. A. (2014). Obesity misclassification and the metabolic syndrome in adults with functional mobility impairments: Nutrition Examination Survey 2003-2006. Preventive Medicine, 60, 71-76. http://doi.org/10.1016/j.ypmed.2013.12.014

Prestes, J., \& Tibana, R. A. (2013). Muscular static strength test performance and health: absolute or relative values? Revista da Associação Médica Brasileira, 59(4), 308-309. http://doi.org/10.1016/j.ramb.2013.01.009

Roohafza, H., Sadeghi, M., Talaei, M., Pourmoghaddas, Z., \& Sarrafzadegan, N. (2012). Psychological status and quality of life in relation to the metabolic syndrome: Isfahan Cohort Study. International Journal of Endocrinology, 2012, 380902. http://doi.org/10.1155/2012/380902

Rubim, V. S. M., Drumond Neto, C., Romeo, J. L. M., \& Montera, M. W. (2006). [Prognostic value of the Six-Minute Walk Test in heart failure]. Arquivos Brasileiros De Cardiologia, 86(2), 120-125. http://doi.org//S0066782X2006000200007

Sarrafzadegan, N., Gharipour, M., Ramezani, M. A., Rabiei, K., Zolfaghar, B., Tavassoli, A. A., ... Yousefi, A. (2011). Metabolic syndrome and health-related quality of life in Iranian population. Journal of Research in Medical Sciences: The Official Journal of Isfahan University of Medical Sciences, 16(3), 254-261.

Schenkeveld, L., Pedersen, S. S., van Nierop, J. W. I., Lenzen, M. J., de Jaegere, P. P. T., Serruys, P. W., \& van Domburg, R. T. (2010). Healthrelated quality of life and long-term mortality in patients treated with percutaneous coronary intervention. American Heart Journal, 159(3), 471-476. http://doi.org/10.1016/j.ahj.2009.12.012

Schultz, A. B., \& Edington, D. W. (2009). Metabolic syndrome in a workplace: prevalence, comorbidities, and economic impact. Metabolic Syndrome and Related Disorders, 7(5), 459468. http://doi.org/10.1089/met.2009.0008 
Sociedade Brasileira de Cardiologia. (2010). VI Diretrizes Brasileiras de Hipertensão. Arquivos Brasileiros de Cardiologia, 95(1), 1-51. http://doi.org/10.1590/S0066$782 \mathrm{X} 2010001700001$

The World Health Organization Quality of Life assessment. (1995). The World Health Organization Quality of Life assessment (WHOQOL): position paper from the World Health Organization. Social Science \& Medicine (1982), 41(10), 1403-1409.

Tibana, R. A., Balsamo, S., \& Prestes, J. (2011). Associação entre a força muscular relativa e pressão arterial de repouso em mulheres sedentárias. Revista Brasileira de Cardiologia, 24(3), 163-1698.

Tibana, R. A., \& Prestes, J. (2013). Treinamento de Força e Síndrome Metabólica: uma revisão sistemática. Revista Brasileira de Cardiologia, 26(1), 66-76.

Tibana, R. A., Tajra, V., César, D., Farias, D. L. de, Teixeira, T. G., \& Prestes, J. (2011). Comparação da força muscular entre mulheres brasileiras com e sem síndrome metabólica. ConScientiae Saúde, 10(4), 708-714. http://doi.org/10.5585/ConsSaude.v10i4.3017

Tibana, R. A., Teixeira, T. G., Farias, D. L. de, Silva, A. de O., Madrid, B., Vieira, A., ... Prestes, J. (2012). Relation of neck circumference and relative muscle strength and cardiovascular risk factors in sedentary women. Einstein (São Paulo), 10(3), 329-334.
Van Gaal, L. F., Mertens, I. L., \& De Block, C. E. (2006). Mechanisms linking obesity with cardiovascular disease. Nature, 444(7121), 875880. http://doi.org/10.1038/nature05487

Vieira, D. C. L., Tibana, R. A., Tajra, V., Nascimento, D. da C., de Farias, D. L., Silva, A. de O., ... Prestes, J. (2013). Decreased functional capacity and muscle strength in elderly women with metabolic syndrome. Clinical Interventions in Aging, 8, 1377-1386. http://doi.org/10.2147/CIA.S50333

Wijndaele, K., Duvigneaud, N., Matton, L., Duquet, W., Thomis, M., Beunen, G., ... Philippaerts, R. M. (2007). Muscular strength, aerobic fitness, and metabolic syndrome risk in Flemish adults. Medicine and Science in Sports and $E_{X}$ ercise, 39(2), 233-240. http://doi.org/10.1249/01.mss.0000247003.3 2589.a6

Yorgancioglu, A., Havlucu, Y., Celik, P., Dinc, G., \& Saka, A. (2010). Relation between quality of life and morbidity and mortality in COPD patients: Two-year follow-up study. COPD, 7(4), 248-253. http://doi.org/10.3109/15412555.2010.49681 6

Zhuo, Q., Yang, W., Chen, J., \& Wang, Y. (2012). Metabolic syndrome meets osteoarthritis. $\mathrm{Na}$ ture Reviews. Rheumatology, 8(12), 729-737. http://doi.org/10.1038/nrrheum.2012.135

Todo o conteúdo da revista Motricidade está licenciado sob a Creative Commons, exceto quando especificado em contrário e nos conteúdos retirados de outras fontes bibliográficas. 